www.jmscr.igmpublication.org

Impact Factor 5.84

Index Copernicus Value: 71.58

ISSN (e)-2347-176x ISSN (p) 2455-0450

crossref DOI:_https://dx.doi.org/10.18535/jmscr/v5i11.107

Journal Of Medical Science And Clinical Research

\title{
The Functional Outcome of Transtibial vs Transportal Femur Drilling Techniques in Arthroscopic Assisted Anterior Cruciate Ligament Reconstruction
}

\author{
Authors \\ S.Sankar ${ }^{1}$, A.Manikandarajan ${ }^{2}$, P.S.Balamurugavel ${ }^{3}$, Dhanpal Singh ${ }^{4}$, \\ R.Neelakrishnan ${ }^{5}$ \\ ${ }^{* 1}$ Postgraduate in Orthopaedics, RMMCH, Annamalai University \\ ${ }^{2,3}$ Lectutrer, Department of Orthopaedics, RMMCH, Annamalai University \\ ${ }^{4}$ Professor of orthopaedics, RMMCH, Annamalai University \\ ${ }^{5}$ Professor \& Head of Department, Orthopaedics, RMMCH, Annamalai University
}

\begin{abstract}
Aim: Femoral tunnel drilling in ACL reconstruction is done by either anteromedial portal (AMP) or by transportal technique. We compared the functional outcome following arthroscopic assisted ACL reconstruction by transtibial and transportal techniques.

Materials and Methods: From June 2015 to June 2017 a consecutive series of patients with mean age of 30.7 years who underwent ACL reconstruction were included for the study. The femoral tunnelling was done either by anteromedial portal or transtibial technique. The reconstruction was done by either patellar tendon bone graft or quadruple hamstring autograft. Both groups were compared in 8 follows.

Results: The functional results were scored according to the subjective IKDC scoring system and compared by using two separate sample T-test and accordingly the P value came to be less than 0.05.The position of femoral tunnel, ease of technique and pitfalls were analysed.

Conclusion: Anteromedial portal technique shows significantly less mean value of subjective IKDC score on follow ups when compared to transtibial technique. More horizontal placement of femoral tunnel was possible with AMP technique.
\end{abstract}

Keywords: Anterior cruciate ligament tear, anteromedial portal, transtibial.

\section{Introduction}

Anterior cruciate ligament is one of the most commonly injured knee ligaments. Despite all discrepancies in ACL reconstruction, techniques of femoral drilling are still the most important discussion in arthroscopically assisted ACL reconstruction ${ }^{(1)}$.Currently the two main methods used by surgeons are transtibial and anteromedial portal techniques ${ }^{(2)}$.
Still 1990 following ACL injuries ACL reconstruction was the only aim and short term follow ups shows good results in $90 \%$ but long term follow up shows some complication in the form of persistant instability, rerupture, impingement and degenerative changes ${ }^{(3)}$ on careful evaluation, complication are found to be related to the inaccurate femoral tunnel placement in $10 \%-40 \%$ cases $^{(3)}$. 
Hardin et al described femoral tunnel drilling through trastibial technique and Batsoni et al described anteromedial portal technique for the first time in $1998^{(4,5)}$. Conventional transtibial femoral tunnel is inevitabily affected by angle and position of the tibial tunnel, a more vertical femoral tunnel ${ }^{(4,5)}$ and hence in the past decade many investigators have advocated preparation of the femoral tunnel independently through an anteromedial portal, thereby avoiding the constraints of tibial tunnel drilling ${ }^{(6)}$. In this study, comparing the functional and clinical outcomes of anteromedial portal and transtibial technique using either BPTB graft or hamstring graft was done. IKDC scoring system was used.

\section{Materials and Method}

Study was conducted from June 2015 to June 2017. Written informed consent from the patients undergoing the study was taken. All patients who underwent unilateral arthroscopically assisted ACL reconstruction using $\mathrm{BPTB}$ or hamstring graft were include in the study.

\section{Inclusion criteria}

History of giving way, positive grade III lachmans test, conformation of ACL tear by MRI imaging and 3 weeks to 6 months interval between injury and surgery.

\section{Exclusion criteria}

Patient having rupture of other knee ligaments, meniscal injury, arthritic changes, previous knee injury and native management.

In our study patient age between18-45. Finally from 60 qualified patients, 30 patients were in the transtibial and 30 patients were in the anteromedial portal group. IKDC scoring was performed on respective follow ups.

\section{Surgical Techniques}

Diagnostic arthroscopy was done with standard anterolateral portal and confirmed ACL tear. Bone patella tendon bone graft or hamstring graft was harvested and prepared. Medial working portal was made, in trastibial method, after flexing the knee upto $90^{*}$ tibial jig is inserted through the anteromedial port. Guide wire is inserted with the help of jig in both tibia and femur, appropriate reamers to graft diameter were used to make tibial and femoral in 11" o clock and 1"o clock position for right and left respectively. In AMP technique using an anteromedial portal, as the knee was flexed more than 90 degree and femoral tunnel was positioned and created in 9 to 10 " o clock position for the right and 2 to 3 "o clock for the left knee joint. Graft fixation was done by titanium interferential in both tibial and femoral sides. In all the cases the diameter of applied screws were selected based on the graft diameter

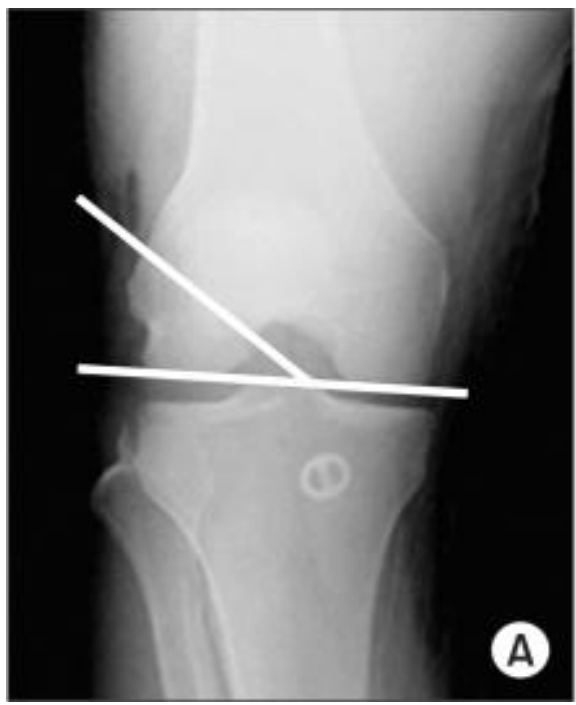

Transportal

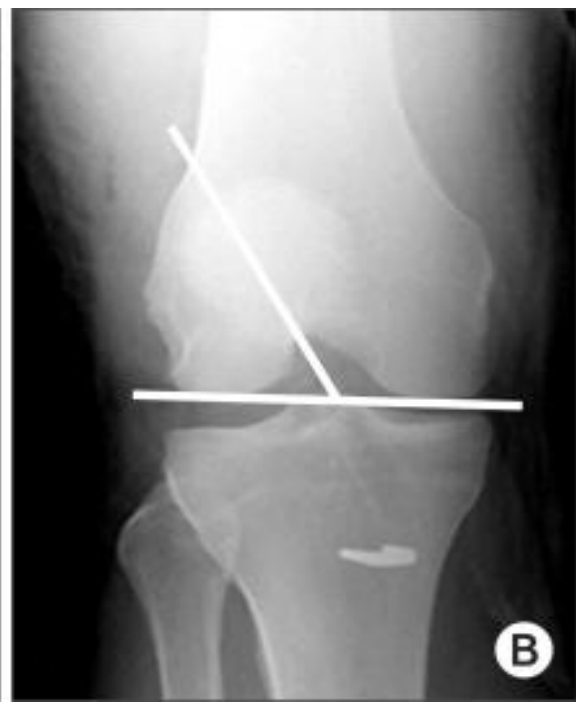

Transtibial 


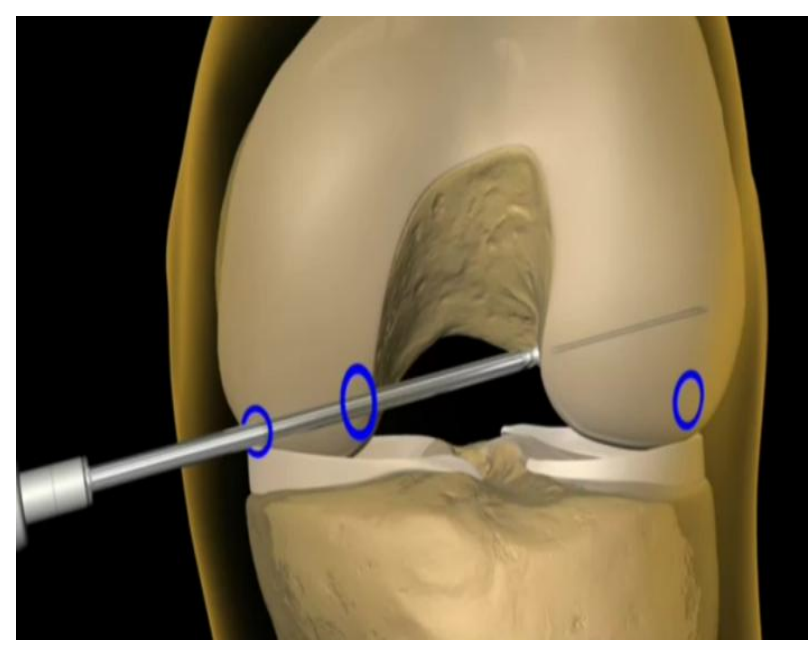

Transportal Femoral Tunnel Drilling

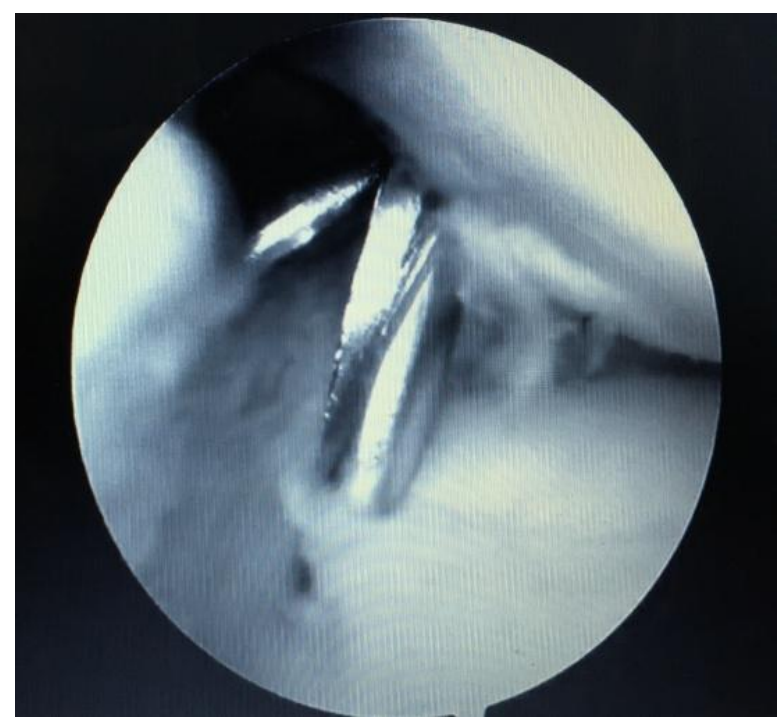

Transtibial Tunnel Placement

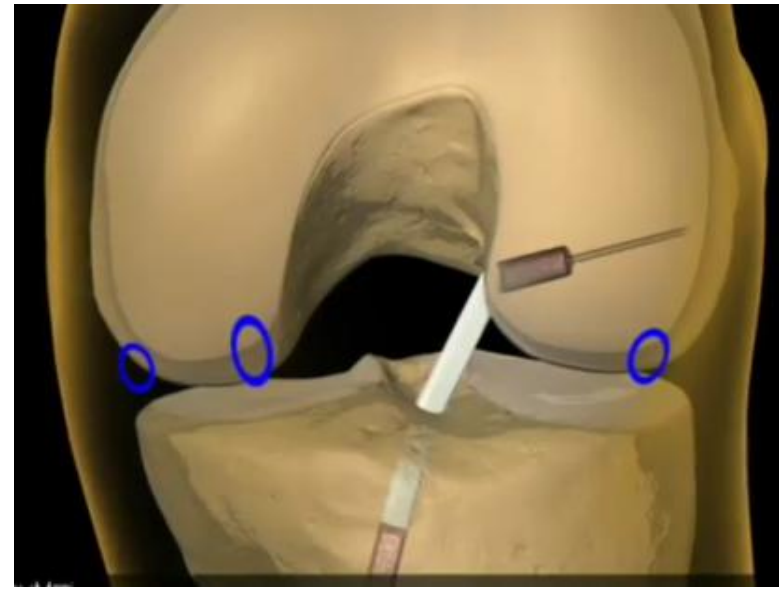

Transportal Graft Placement

\section{Post-OP Rehabilitation}

In both techniques patients were placed in long knee brace and compression bandage was applied and 4 doses of $3^{\text {rd }}$ generation cephalosporin was given. Post operatively drain tube removed by 48 hours and sutures removed by 12 days. Weight bearing stared by immediate post operative period but knee flexion allowed after 3 weeks and 
jogging was permitted at $4^{\text {th }}$ month. In between period patient were encouraged to do static quadriceps exercise. In our study patients were followed up at 8 intervals. Functional outcome was determined by using the subjective IKDC scoring system from the immediate post operative period to the patient returning to the normal daily activities.

\section{Results}

In our study, all 60 observed cases, 45 patients were male $(85 \%)$ and 15 were $(15 \%)$ females. The mean age of the patients 30.7. Patients age and sex distribution was not considerably different in the two observed groups. Results were studied according to the IKDC scoring system, by using two independent sample T-test, the $\mathrm{P}$ value were found to be $<0.05$ at all intervals. The $\mathrm{P}$ value < 0.05 suggest that there is a significant difference between the functional outcome of the two technique and there is better anatomical graft placement and better rotational stability when compare to the transtibial technique. Anteroposterior translation of reconstructed ligaments in both the techniques was assessed by lachman test, which showed significant changes in transtibial technique compare to anteromedial technique. (I.e. laxity is more in transtibial compared to anteromedial portal in short term follow up), which is not present in long term follow up. None of the patient had poor result in both transtibial and anteromedial portal technique.

TRANSTIBIAL

X RAY \& MIRI LEFT KNEE

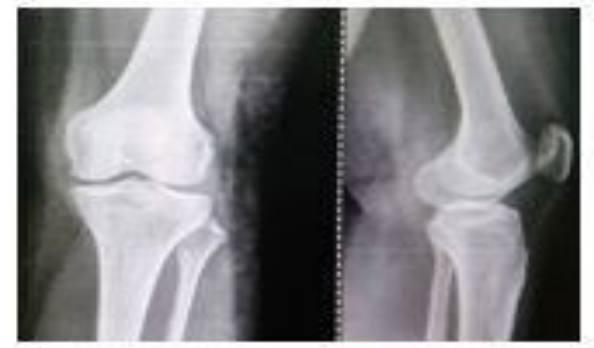

PRE OP PICTURE

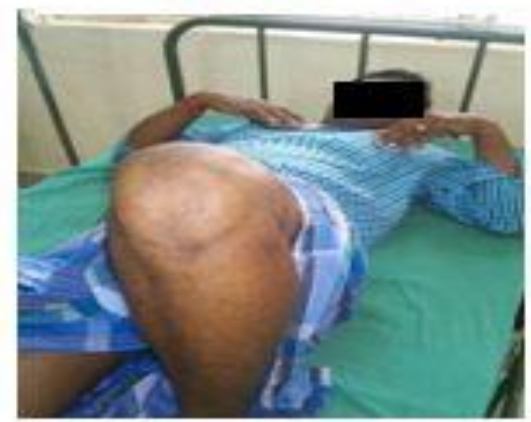

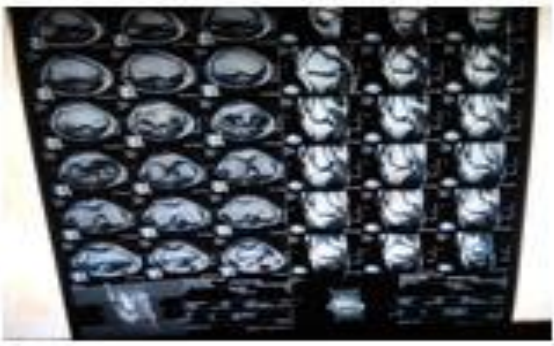

POST OP X-RAY

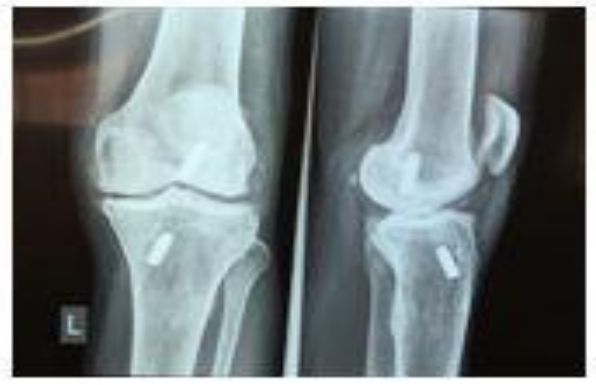

6 MONTHS POST OP PICTURES
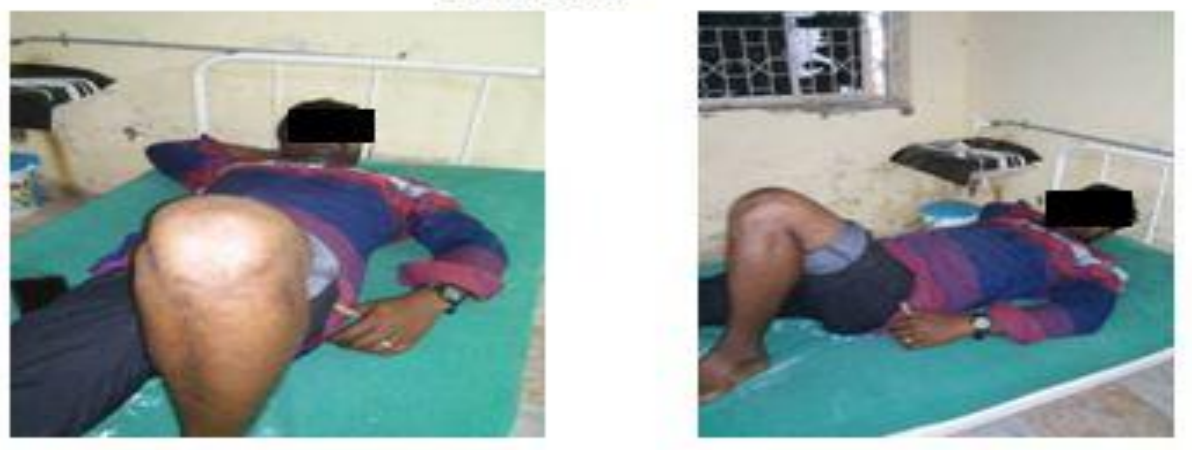
TRANSPORTAL

PRE OP X RAY \& MRI RIGHT KNEE

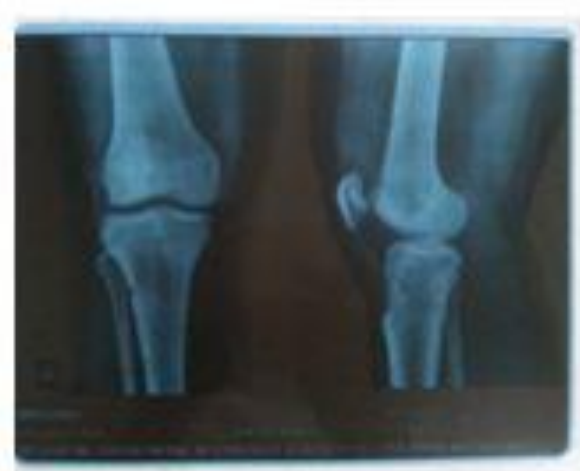

PRE OP PICTURE

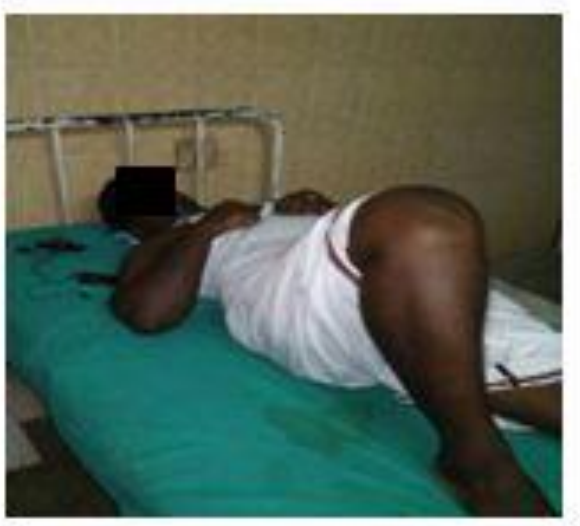

2 MONTHS POST OP PICTURE

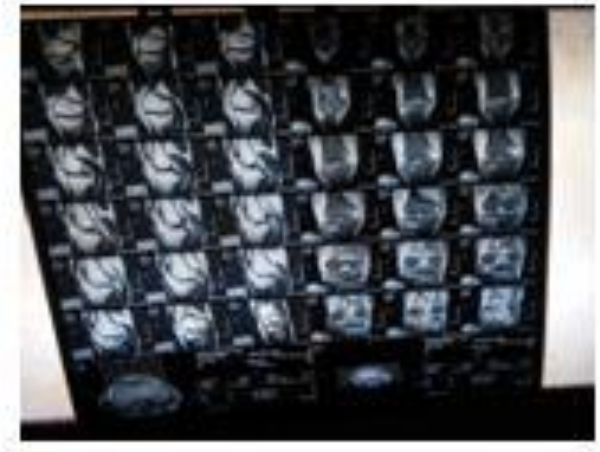

POST OP X-RAY

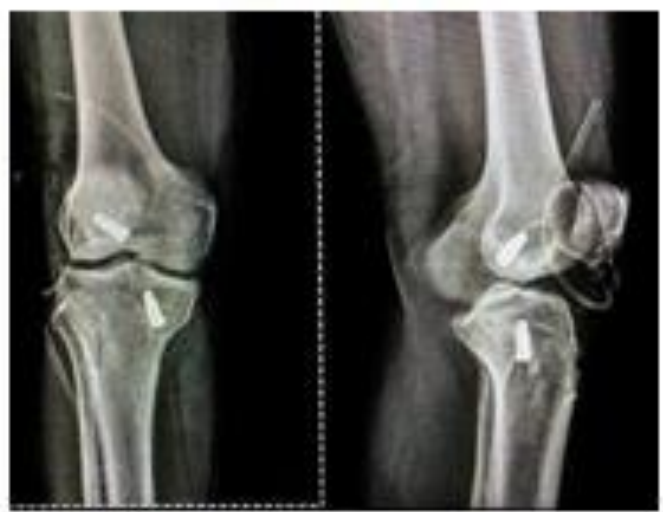

5 MONTHS POST OP PICTURES
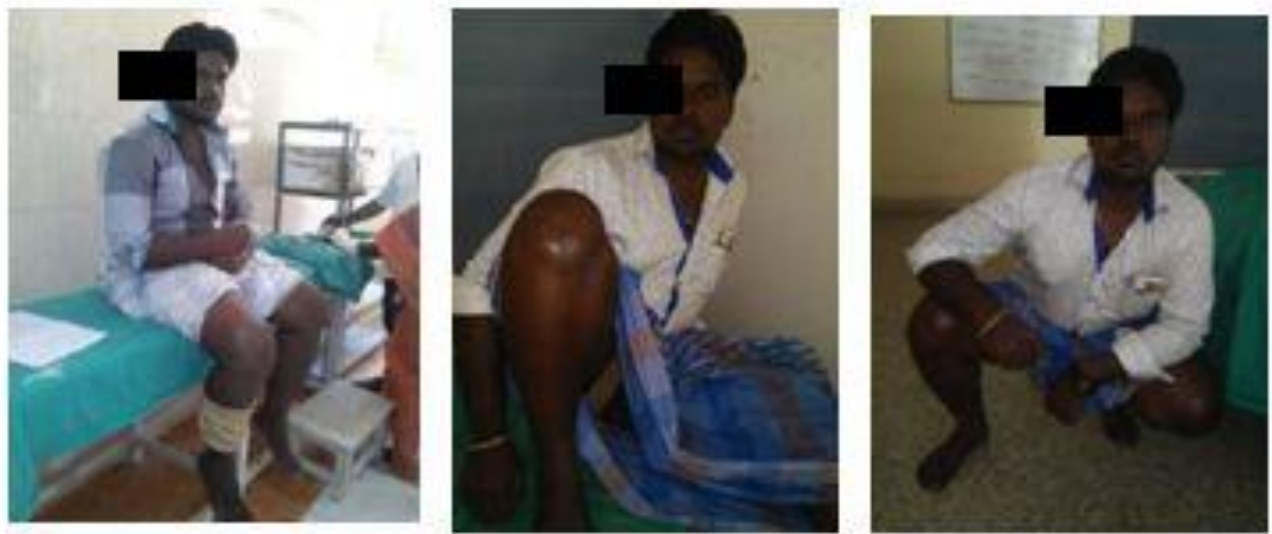

\section{Discussion}

In this study, we analysed the clinical outcome of AMP and transtibial technique of femoral tunnel drilling arthroscopic ACL reconstruction. Various advantages and disadvantages of transtibial technique are known. It is technically simple, no accessory portal is required and may need less time.However vertical placement of ligament is common with transtibial technique which leads to rotational instability and higher incidence of major drawbacks ${ }^{(4,6)}$. AMP has advantage of more oblique femoral tunnel, thus reducing the chance of rotational instability. It has better chance of achieving a more anatomical femoral tunnel ${ }^{(4,6)}$, but there are some rare complications like guide wire breakage during hyperflexion of knee joint, 
posterior cortex blowout ${ }^{(4)}$. In our study there were no intraoperative complication was noted. Anteromedial portal approach has certain disadvantages in terms of short femoral canal which might affect the fixation modality and graft healing and this complication also not noted in our study. This may explain the anteromedial portal group quicker return to routine activities ${ }^{(1,4)}$. Considered knee laxity, studies with short term follow up confirmed obvious advantage of anteromedial portal compared to transtibial, but again in long term follow ups this laxity was disappeared ${ }^{(1,4)}$. The anterior and posterior laxity of the knee in transtibial may be because of the femoral graft is placed in a more anterior position than in AMP. Alentorn- geli in a similar study with BPTB graft recently conducted that AMP technique prominently restores higher stability in rotational and antero-posterior movements ${ }^{(4)}$. Heming et al concluded that the transtibial has the capability of femoral drilling but it may lead to disproportion tunnel length- tendon length (4), although most recent papers have supported the anteromedialportal method when compared to the transtibial technique should not be abandoned just because of the results of this and a few other studies.

In our prospective study, we used subjective IKDC scoring system, which is to be consistently better in anteromedial portal group at all follow up intervals.

It should be acknowledged that one of the limitations of this study was non availability of KT -1000 , so clinical examination in our study was only subjective. Short term follow up study should be mentioned another one limitation. Both of groups were not completely homogenous and were not matched in terms of type of sport or type of work and daily activity. However with regards to the 2 years follow up of the study, the above mentioned variables would not particularly impact the functional outcome of the study. Major strength of the present study is that it reports the experience of a single centre, single surgeon and similar rehabilitation program.

\section{Conclusion}

Both anteromedial portal and transtibial drilling technique in arthroscopic assisted ACL reconstruction showed similar results in clinical outcome according to subjective IKDC knee scoring system. Still anteromedial portal technique have advantage of femoral tunnel drilling in more horizontal placement which is the anatomical position.Ease of femoral interferential screw placement in anteromedial portal than transtibial technique.

\section{References}

1. Alentorn - Geli E, lajara F,Samitier G et al:the transtibialverus the antromedial portal technique in the arthroscopic bone patellar tendon -bone anterior cruciate ligament reconstruction. Knee surg sports traumatol artrosc,2010;18:1013-37.

2. Duquin TR, Wind WM, Fineberg MS et al: currenttrend in anterior cruciate ligament reconstruction. J knee surg, 2009:22:7-12.

3. Anderson AF, Snder RB, Lipscomb B Jr.Anterior cruciate ligament reconstruction .A prospective randamized study of three surgical methods. Are J sports med 2001; 29:272-279.

4. Anteromedial portal vs transtibial techniques for drilling femoral tunnel in ACL reconstruction using 4-strand hamstring tendon: ac cross sectional study with I year follow up.

5. Bottoni CR, Rooney RC, harpstrite JK et al: Ensuring accurete femoral guide pin placement in anterior cruciate ligament reconstruction. AMJ orthop, 1998:27:76499

6. Howell SM, clark JA: Tibial tunnel placement in anterior cruciate ligament reconstruction and graft impingement. Clinorthorelat res.1992 Oct; (283): 18795. 